\title{
The High-Pass Filter Hypothesis for Autism Spectrum Disorder
}

\author{
Luca DellAnna
}

\begin{abstract}
This paper proposes that one of the underlying causes of Autism Spectrum Disorder is the presence of a High-Pass Filter Effect applied to cognitive perception. Such an effect causes an enhancement of local details at the expense of global patterns. In particular, the High-Pass Filter Effect induces a disadvantage pertaining to mastering contextual fields (fields in which local features are noisy and information is distributed in adjacent perceptual pieces and in the relationship between them; for instance, interpersonal communication). Furthermore, in some cases, the High-Pass Filter Effect produces an advantage with respect to mastering detailed fields (fields in which information is intrinsic in modular, precise pieces of information; for example, computer science).

In this paper, the High-Pass Filter Effect is explained, two secondary effects are described, (Peripheral Functionality Blindness and Prioritization by Specificity), and then a few examples of the way in which these effects induce the symptoms of Autism Spectrum Disorder are reported.
\end{abstract}

\section{Introduction}

I hypothesize that at least a few of the cognitive properties of Autism Spectrum Disorder (ASD) are caused by the presence of a High-Pass Filter Effect applied to perception. "High-pass filter" refers to a filter that enhances the fine details and reduces the broader patterns (I do not use the term with reference to its strictly electronic meaning, "filter that blocks low frequencies". Rather, I refer to a neurological equivalent that produces transformations similar to the ones that an electronic high-pass filter would create). The farther an individual is on the Autism Spectrum, the stronger the effect of such a filter. In the first section of this paper, "The World Through a Magnifying Glass", I provide a visual example to clarify the way in which the High-Pass Filter Effect is perceived in practice.

This effect has two consequences, which are as follows: more details are relayed to the higher perceptual areas of the brain, and perception is examined in a more focused and less contextual manner.

The fact that a high-pass filter enhances local features at the expense of global patterns causes the people on the Spectrum to consider only the "central features" and ignore the "peripheral features". This places them at a disadvantage to learn any field where such peripheral features are important (body language and facial expressions are examples of the features of interpersonal communication that are peripheral but important; words would constitute the central feature).

The fact that this filter relays a higher quantity of enhanced details to perception causes people with ASD to organize concepts into narrower categories with more distinct boundaries. The more details one perceives, the more categories she/he constructs with respect to which one should classify a given class of objects. This allows them to better analyze precise, complex systems. On the other hand, this makes him them vulnerable to noise (for reasons that will be examined in greater detail below). Consequently, it presents a disadvantage with regard to understanding noisy fields, such as human behavior or motor coordination.

The aforementioned concepts will be explained in depth in the remaining part of this paper, which is a technical extract from my last book, "The World Through a Magnifying Glass: The High-Pass Filter Hypothesis for Autism Spectrum Disorder".

\section{The World Through a Magnifying Glass}

This section elucidates the perceptual effect of a high-pass filter in an extremely visual manner. The following sections will provide a technical description of the High-Pass Filter Effect, whereas this one is required to facilitate an intuitive understanding of the relevant concepts. (Booth \& Happé, 2010) first proposed the sentence in the example, as a test for Central Coherence.

Imagine reading a book with a magnifying glass. If the magnifying power of the lens is low, one complete sentence can be read at a time (Figure 1). You can effortlessly focus on the meaning of the sentence as a whole. Moreover, you can easily predict the subsequent words. In the example provided in Figure 1, the phrase "You can go hunting with a knife and" can easily be completed by guessing the phrase "catch a bear". This is the way in which a person who is not on the Autism Spectrum (a Neurotypical) perceives the world. 


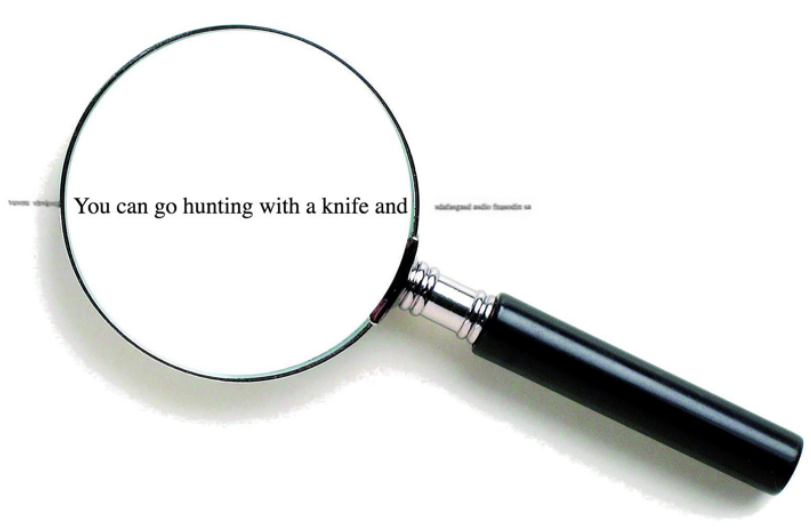

Figure 1. How a Neurotypical perceives the world

Now, imagine reading a book using a stronger magnifying glass; this time, you can only read a couple of words at once (Figure 2). You start reading from the beginning of the sentence, a few words at a time, until you reach the phrase "a knife and". At this point, your mind, focused on the few words it is seeing, will be tempted to predict the following ones as "a fork". The complete sentence, "You can go hunting with a knife and a fork", does not make much sense. However, if you consider the point that you could only see the phrase "a knife and", the "a fork" completion seems to be suitable. After all, "a knife and a fork" is a more common pattern compared to the phrase "a knife and catch a bear". This is the way in which a high-functional autistic individual perceives the world. It is already known that ASD is associated with a deficit pertaining to one's ability to broaden the spatial spread of visual attention (Mann \& Walker, 2003) (in the above-stated example, this point corresponds to that of the lens showing fewer letters at a time).

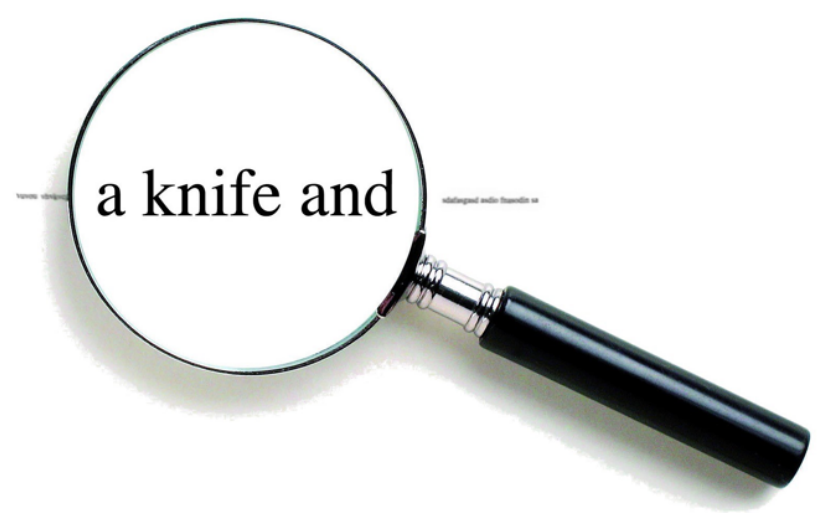

Figure 2. How a high-functional autistic individual perceives the world

Lastly, imagine reading with an extremely powerful magnifying glass, whose zooming effect is so strong that it only allows you to view a few letters at a time (Figure 3). You are unable to make use of the global meaning of the complete sentence anymore; you cannot use the context provided by the words preceding "and" (words that you cannot see any longer) to predict what will follow. Instead, the few characters that you can see at a time appear much more prominently in front of your eyes, and other thoughts start to occupy your mind (for example, the font used to write them). These thoughts prevent you from memorizing the sentence word by word, in order to try and analyze its meaning as a whole. Consequently, you are stuck trying to determine the meaning of each word in isolation and the way in which they are written (for instance, why did they use this font? Why is that small ink stain there? What does that ink stain remind me of?). This is the way in which a low-functional autistic person perceives the world.

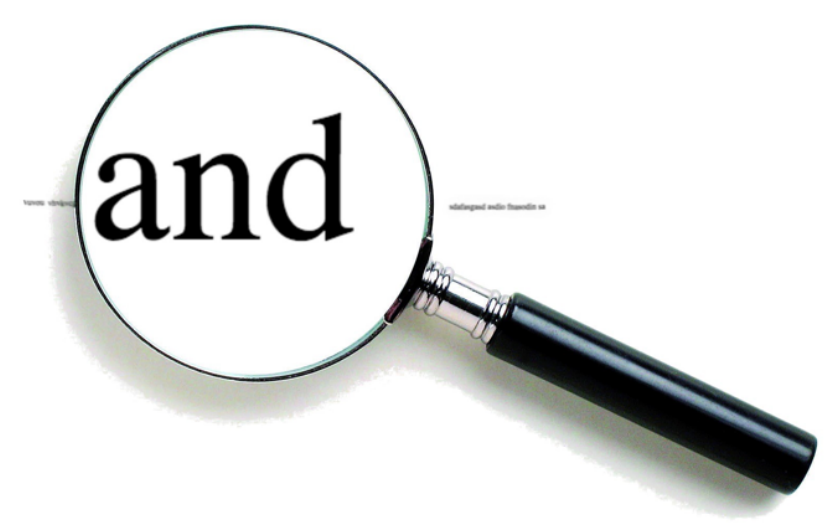

Figure 3. How a low-functional autistic individual perceives the world

The Magnifying Glass effect explains the numerous effects that were previously collectively referred to as "Weak Central Coherence"; how can a person maintain central coherence if she/he is only able to focus on a single, restricted scope at a time?

\section{The High-Pass Filter Effect}

The general definition of a high-pass filter, as taught during electronics classes, is 'a filter that lets narrow and local changes pass through and blocks broader patterns instead.' Such a perceptual filter helps people better perceive local variations (details) at the expense of overlooking peripheral features (context). I postulate that each individual, including Neurotypical ones, possesses a bandpass filter. However, its intensity (how much a small detail is enhanced) and bandpass (how small a detail must be in order to be enhanced) are the qualities that determine whether, and how farther, a person is placed on the Autism Spectrum.

The High-Pass Filter Effect explains all the experimental re- 
sults in which the people on the Spectrum demonstrated a preference for high-frequency features. For instance, it has been reported that people on the Spectrum tend to rely on high-frequency features in order to analyze faces (Deruelle, Rondan, Gepner, \& Tardif, 2004).

A high-pass filter does not only causes an atypical perception of those features that are on the frequency dimension, but it works with respect to many dimensions: visual detail, colors, object features, word meanings, and so on. The best way to imagine the effect of a high-pass filter is to consider a filter that examines each little piece of perception and compares it with the adjacent ones; if it is different with regard to any salient dimension, it applies a gain factor to the perceptual piece. However, this comes at the expense of overlooking global patterns, that is, information distributed on the more proximate pieces of perceptual information.

Please regard the example provided in the table in Figure 4. The first line presents a random sequence of numbers, whereas the second and third lines represent the effect of filtering the first sequence through a high-pass filter as well as a low-pass one, whose equations are reported the table provided below (Figures 5 and 6).

\begin{tabular}{lccccccccccccc} 
Time & 0 & 1 & 2 & 3 & 4 & 5 & 6 & 7 & 8 & 9 & 10 & 11 & 12 \\
Input sequence & 2,00 & 2,00 & 2,00 & 2,00 & 3,00 & 4,00 & 7,00 & 5,00 & 3,00 & 2,00 & 2,00 & 2,00 & 2,00 \\
Sequence after high-pass filter & 2,00 & 2,00 & 2,00 & 1,75 & 3,00 & 3,50 & 8,25 & 5,00 & 2,75 & 1,75 & 2,00 & 2,00 & 2,00 \\
\hline Sequence after low-pass filter & 2,00 & 2,00 & 2,00 & 2,25 & 3,00 & 4,50 & 5,75 & 5,00 & 3,25 & 2,25 & 2,00 & 2,00 & 2,00
\end{tabular} Figure 4. The input sequence and the two output sequences generated by the two filters

$$
y\left(x_{i}\right)=-\frac{1}{4} x_{i}-1+\frac{3}{2} x-1-\frac{1}{4} x_{i}+1
$$

Figure 5. An example of a high-pass filter

$$
y\left(x_{i}\right)=+\frac{1}{4} x_{i}-1+\frac{1}{2} x-1+\frac{1}{4} x_{i}+1
$$

Figure 6. An example of a low-pass filter

In the graph included in Figure 7, by observing the dotted line only (the sequence passed through the low-pass filter), a general upward trend in the first part of the sequence coupled with a general, gradual downward trend in the second part are noticeable. Alternatively, by observing the solid line (the sequence passed through the high-pass filter), we can notice a single bump in the middle of the sequence (greater perception of detail), but no gradual increase (lesser perception of trend) is observed. Therefore, the high-pass filter di-

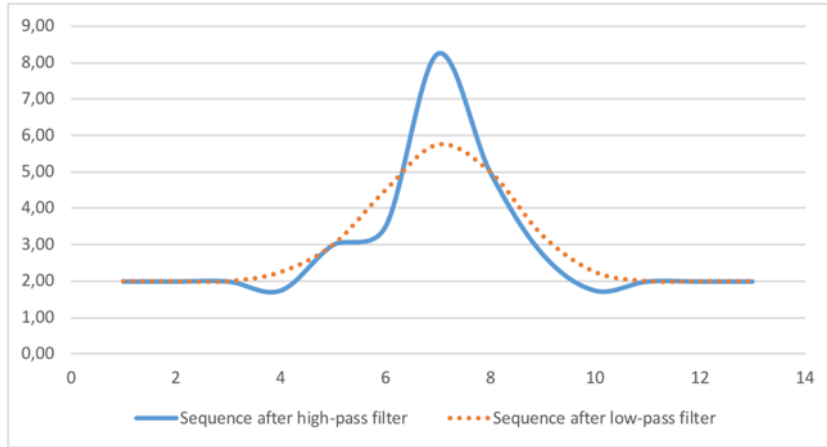

Figure 7. A graph of the two output sequences

rected our focus toward a local point (the bump) while underemphasizing the broader features. Hence, it can be asserted that high-pass filters divert our focus toward the local details, while overlooking the broader pattern. High-pass filters induce what has been referred to as Weak Central Coherence. Furthermore, I need to stress on the point that the High-Pass Filter Effect applies not only to sequences but also to all kind of patterns, as it will be established in the following sections of this paper, which will cover practical examples of the way in which this effect applies to ASD. For instance, it might be applicable in the case of a face-to-face conversation, focusing on the local (e.g., the specific meaning of each spoken word) rather than the global aspect (e.g., the body language, or the fact that a conversation is not only about spoken words but also about body language and tone of voice).

\section{The causes of the High-Pass Filter Effect}

The underlying causes of the High-Pass Filter Effect still remain unclear. I have provided a hypothesis based on the columnar structure of the brain in (DellAnna, 2018) and in my book "The World Through a Magnifying Glass: The High-Pass Filter Hypothesis for Autism Spectrum Disorder".

\section{Peripheral Functionality Blindness}

The people who are on the Spectrum are oblivious to the aspects that are not at the focus of the Magnifying Glass. They only perceive the pieces of information positioned in its center, at the focus, but they are blind to the ones that are available on its periphery. The same phenomenon occurs with respect to the purposes, or functions, of human activities. Most activities possess a central purpose (the publicly stated one) and one or more peripheral purposes (the actual reasons for Neurotypicals caring about that particular activity) (Hanson \& Simler, 2017).

I propose that the people on the Spectrum are oblivious to such peripheral functions of activities and are therefore less proficient at most activities whose real purpose is different 
from the stated one. A few examples of such activities are as follows:

- Small talk (it is not about what is discussed but about evaluating the conversation's partner and building trust

- Romantic gestures (it is not about the gesture itself but about showing care and commitment)

- Dressing (it is not about protecting oneself from the cold and covering one's intimate parts but about signaling values)

- Play (it is not about the game itself but about social bonding)

- Religion (it is not about whether the beliefs are true but about a sense of purpose, spirituality, community, and earning social capital); typically, the people on the Spectrum are more likely to identify as atheists or agnostics (Caldwell-Harris, Murphy, Velazquez, \& McNamara, 2011).

A note to all the Neurotypicals reading this section: it does not matter if the points in the aforementioned list are obvious to you and to everyone else you are acquainted with. It might not be obvious to a person on the Spectrum.

In general, the people on the Spectrum tend to ignore the unstated purposes of activities to focus on the stated ones, even if the former are more important or evident (to Neurotypicals). They undertake activities as though the only factor that mattered was its stated function. Therefore, to Neurotypicals, they might appear to be acting weirdly or inappropriately (vice versa, Neurotypicals could appear to be acting weirdly or pointlessly according to the people on the Spectrum).

\section{Prioritization by Specificity}

Imagine you are a person on the Spectrum. You are listening to someone talking to you and gather a great deal of information in the process: words, inflections in their voice, facial expressions, body language, and so on. From all of them, you need to extract the meaning that the speaker wants to convey. You are excellent at understanding the meaning of words (you know the very specific meaning of each of them). However, you are very poor at reading facial expressions and body language. Every time you see a person's expression, you are unsure of its meaning. Therefore, if you receive conflicting information from the words spoken as well as the body language, you are much more likely to prioritize the one with lesser incertitude associated with it, which, in this case, are signified by words.

For a Neurotypical, a given facial expression might convey a single meaning. However, for a person on the Spectrum, this is point does not stand true; since they are not proficient at classifying them, they associate a larger distribution of meanings to any given observed expression.

I propose that the people on the Autism Spectrum tend to prioritize information channels whose items have a higher specificity (a lower number of meanings associated with each item, from their own point of view). In particular, this phenomenon causes the people on the Spectrum to over rely on words and ignore other communication channels, such as body language, tone of voice, and facial expressions.

\section{Contextual and Detailed Fields}

High-functioning ASD can either be an advantage or a disadvantage in mastering a given field, depending on whether it is a detailed field or a contextual one respectively. The differences between the two fields are highlighted in Table 1 and clarified below.

\begin{tabular}{|l|l|}
\hline Detailed field & Contextual field \\
\hline Intrinsic informative value & Extrinsic informative value \\
\hline Modular & Integral \\
\hline Precise & Imprecise \\
\hline
\end{tabular}

Table 1

Differences between detailed and contextual fields

Intrinsic / Extrinsic informative value. Pieces of information in detailed domains tend to possess intrinsic informative value; they hold value on their own, and their meaning does not depend on other pieces of information. A mathematical function can be studied on its own. A code block in computer science (such as a function or a class) has its internally defined meaning and behavior. There is no need to view the broader context of their placement, before ascertaining a several aspects pertaining to what they are, what they do, how they can be used, and what constraints they have. Conversely, pieces of information in contextual domains tend to possess extrinsic informative value; their meaning depends on the context they are placed in. The meaning of a conversational sentence can change depending on the context ("I would die for this") or on contextual clues, such as the tone of voice (for example, a sarcastic remark such as "I'm so happy to meet you", if spoken without a smile). Before being able to fully comprehend a piece of information in a contextual domain, one has to regard its context.

The High-Pass Filter Effect prevents the people on the Spectrum from perceiving the broader context. They only see the details concerning what their perception is focused on and cannot view the surrounding area. Therefore, they are impaired with respect to understanding contextual fields; they overlook data that is fundamental for evaluating the few pieces of information that they are focusing on. This is the reason, for example, they tend to take sentences literally. On 
the other hand, the people on the Spectrum are advantaged with regard to mastering detailed fields. They only see the information that matters, and they perceive it precisely.

Modular / Integral. Detailed domains tend to be modular, whereas contextual ones are usually integral. In modular fields, pieces of information can be easily rearranged, without altering their overall functioning. This is not so in the case of integral ones.

Moreover, in modular fields, one can specialize in a single sub-field and be fare quite well; once again, this is not the case with respect to integral ones.

Furthermore, in such fields, one can evaluate the whole by studying a part; in the case of integral ones, this is not possible.

For instance, shooting a basketball is an example of a contextual and integral field. Even though there are many rules pertaining to the way one should shoot the ball, such as, for a right-handed player, "bend your right arm 90 degrees" and "hold your left hand at the side of the ball". However, one could follow all these rules and still be a bad shooter. On the other hand, certain professional basketball players, such as Shawn Marion and Tayshaun Prince, managed to be recognized as good long-range shooters even with extremely poor and technically incorrect shooting techniques. What matters is that the shooting movement as a whole should be coordinated. It is impossible to look at the flexion of a single muscle and determine whether that shot will be a hit or a miss; one must observe the complete movement of the player.

Precise / Imprecise. Contextual fields tend to be imprecise. Since the informative value is not contained in a single, independent item but is rather situational, contextual fields can are relatively insensible to small imprecisions in the items composing them. Conversely, detailed fields rely on each item to provide precise information. Therefore, no imprecision can be tolerated in this regard.

The people on the Spectrum are impaired with respect to learning from contextual fields, as they perceive, process, and store information in a detailed manner, that is, in a precise manner, which is exceedingly sensitive to the noise and imprecision that is characteristic of contextual fields.

\section{Example: Interpersonal communication}

Interpersonal communication is a highly contextual field (unless we consider the most formal conversations). The way in which the points described in the previous sections are applicable in this regard have been presented below.

- Extrinsic value of information: Words cannot be examined individually to determine their meaning; one must also consider the reason they are related to one's tone (for instance, in the case of sarcasm) or body language (for example, in case the speaker is lying).
- Integral field: A person could master the use of words and still be a terrible speaker (for instance, that boring guy at the last conference you attended).

- Imprecise: Unless in the case of an extremely technical conversation (such as a conversation between two scientists), one can misuse a few words and still deliver the message effectively; on the other hand, one could choose very precise words and completely fail to deliver an effective message, if the body language is incoherent.

- Peripheral Functionality Blindness: The people on the Spectrum tend to despise small talk, as they only perceive the most evident function of interpersonal communication, which is exchanging information through spoken words. However, they are blind to the less evident but more important functions of small talk, such as building trust, evaluating the conversation's partner, building social capital, and so on.

- Prioritization by Specificity: High-functioning autistic individuals think that communicating on a single channel is enough (as the High-Pass Filter Effect highlights only one channel at a time). They usually select the one with less subjective uncertainty associated with it (words) and entirely ignore the other factors (body language, facial expressions, to name a few).

\section{Learning impairment for contextual fields}

To demonstrate the way in which contextual fields appear to be noisy to people affected by ASD, a thought experiment involving learning sequences of shapes has been posited.

Let's imagine that a rule exists in the external world stating that two four-sided pieces in a row are always followed by a circle. For instance, let's imagine the following sequence (the shapes written in bold are the ones that adhere to the above-stated rule): rhomboid, square, circle, triangle, square, rhomboid, circle, star, square, star, trapezoid, square, circle. Neurotypicals, who possess a weaker HighPass Filter Effect and therefore tend to make use of less categories, could include rhomboids, squares, and trapezoids (all of which have four sides) in the "squares" category, without recognizing them to be separate sub-categories; their lack pertaining to a High-Pass Filter Effect fails to highlight the differences between these shapes (this is not literally true, of course; it is just an exaggerated metaphor to illustrate the way in which perception works). Hence, a Neurotypical would see the sequence as follows: square, square, circle, triangle, square, square, circle, star, square, star, square, square, circle. It would only take him/her a few seconds to infer the rule that "two squares in a row are always followed by a circle". Conversely, the people affected with ASD, who puts squares, trapezoids, and rhomboids 
in separate categories instead, would see the sequence as follows: rhomboid, square, circle, triangle, square, rhomboid, circle, star, square, star, trapezoid, square, circle. It is much harder for them to notice the aforementioned rule. However, they could report that they inferred a different rule, according to which rhomboids are always followed by a square. This rule is not a rule in reality but a product of randomness in the sequence, which, in other words, signify noise.

Observing the inappropriate level of detail (abundant detail in a contextual field) prevents the people on the Spectrum to learn the broad patterns and leads them to learn false, narrower patterns that are only noise. Therefore, their learning process is impaired.

It must be noted that if the sequence did not represent a noisy field (where the rule is broad, such as two four-sided pieces in a row are always followed by a circle) but a nonnoisy field (where the rule is narrow and specific, such as rhomboids are always followed by a square), then a person affected with ASD, who employs more detailed categories, would be advantaged with respect to the learning process: they would be able to see the details necessary to fully understand the rule. This is one of the reasons people with high-functioning ASD are usually exceedingly proficient in learning non-noisy, detailed fields, such as computer science, and poor at learning noisy contextual fields, such as interpersonal communication.

\section{A full spectrum}

It is possible that the High-Pass Filter Effect, with various strength, is broadly distributed on the entire population; a few people could be affected by a strong High-Pass Filter Effect (and they are diagnosed with ASD), most people could be affected by a very low High-Pass Filter Effect or by no particular kind of effect at all' (Neurotypicals), and a few people could be affected by a Low-Pass Filter Effect.

As much as people with ASD excel in detailed tasks and are deficient in contextual ones, there can be another group of people, "extremely contextual", who perform better than Neurotypicals with regard to contextual tasks and worse than them in detailed ones. In other words, if Neurotypicals represent the zero of the Spectrum and low-functioning autistics are placed at the end of the positive side of the Spectrum, there would also be a negative side that includes extremely contextual individuals: people who exhibit "opposite" symptoms compared to autistic ones. If people with autism are affected by a High-Pass Filter Effect, I postulate that there should be a group of people who are, alternatively, affected by a Low-Pass Filter Effect (while the Neurotypicals are affected by none). Such "extremely contextual" individuals have not been labeled yet as a group affected by a disorder, owing to the fact that this disorder does not affect their social life in a negative manner, and/or their dissimilitude has been considered to be socially acceptable. I presume (but this is a presumption that should be substantiated with tests) that such people would exhibit traits such as extreme extroversion, masterful body language, imprecise written language, deficiency in mastering precise fields (for instance, computer science), superior motor coordination (not necessarily paired with precise technique in case of sports), and, in general, a proficiency with regard to contextual fields (though they might or might not be able to master them due to a possible inadequacy pertaining to their capacity to fully reproduce the precision required to excel and/or owing to a possible lack of focus).

This is only an assumption that should be tested and not an essential part of the High-Pass Filter hypothesis for ASD; another possibility is that the High-Pass Filter Effect might be an extremely skewed distribution affecting only the people who are on one side of the population distribution.

\section{Conclusions}

A High-Pass Filter Effect coherently elucidates most of the symptoms of Autism Spectrum Disorder (ASD). Its core effect, which leads to enhancement of local details at the expense of global patterns, causing people with ASD to be disadvantaged in contextual fields, and its byproducts, such as Peripheral Functionality Blindness and Prioritization by Specificity, collectively create a consistent set of origins for most behaviors attributed to ASD.

The High-Pass Filter hypothesis is examined more extensively in my book "The World Through a Magnifying Glass: The High-Pass Filter Hypothesis for Autism Spectrum Disorder", coupled with a number of ASD-related behaviors that have been thoroughly explained, along with a few suggestions regarding the way in which one can facilitate the learning of contextual fields with respect to the people affected by the disorder.

\section{References}

Booth, R., \& Happé, F. (2010). Hunting with a knife and... a fork: : Examining central coherence in autism, attention deficit/hyperactivity disorder, and typical development with a linguistic task. Journal of Experimental Child Psychology(107).

Caldwell-Harris, C., Murphy, C. F., Velazquez, T., \& McNamara, P. (2011). Religious belief systems of persons with high functioning autism. Proceedings of the Annual Meeting of the Cognitive Science Society, 33(33).

DellAnna, L. (2018). The columnar hypothesis for the high-pass filter effect.

Deruelle, C., Rondan, C., Gepner, B., \& Tardif, C. (2004). Spatial frequency and face processing in children with autism and asperger syndrome. Journal of Autism and Developmental Disorders(34). 
Hanson, R., \& Simler, K. (2017). The elephant brain.

Mann, T. A., \& Walker, P. (2003). Autism and a deficit in broadening the spread of visual attention. Journal of Child Psychology and Psychiatry(44). 